\title{
Photonic Band-Gap Inhibition of Modulational Instabilities
}

\author{
Damià Gomila, Roberta Zambrini, and Gian-Luca Oppo \\ Department of Physics, University of Strathclyde, 107 Rottenrow East, Glasgow, G4 ONG, United Kingdom
}

(Received 31 October 2003; published 24 June 2004)

\begin{abstract}
Spatial structures as a result of a modulational instability are studied in a nonlinear cavity with a photonic crystal. The interaction of the modulated refractive index with the nonlinearity inhibits the instability via the creation of a photonic band gap. A novel mechanism of light localization due to defects and pattern inhibition is also described.
\end{abstract}

DOI: 10.1103/PhysRevLett.92.253904

The investigation of spontaneous spatial structures in nonlinear systems is a fast expanding research area, not just in physics but also across science and technology [1]. In particular, spatially extended dissipative nonlinear optical systems display, through self-organization and control, a large variety of outputs and structures with potential applications in photonics, such as optical memories, multiplexing, optical processing, and imaging [2].

There has also been a lot of scientific interest in photonic crystals [3] since the existence of band gaps in such structured materials was predicted by Yablonovitch and John in 1987 [4]. Their unique way of controlling light has provided the field of photonics with new applications, mostly related to guided light modes [5].

More recently, nonlinear effects in photonic crystals were shown to play an important role in achieving alloptical operations in switching devices [6,7]. Transverse effects have been mainly studied in propagation in fiber Bragg gratings within the coupled-mode theory or in infinite coupled arrays of waveguides within the tightbinding approximation. Studies on grating devices focus on the so-called gap (or Bragg) solitons [8], which are pulses with frequencies in the forbidden gaps and extended over a large number of grating periods. In arrays of waveguides, two remarkable features are the formation of the so-called discrete solitons and the fact that the (discrete) diffraction can be engineered [7,9]. Finally, modulation instability shifts due to periodically modulated media have been studied in conservative systems $[8,10]$.

Despite the considerable potential of (nonlinear) photonic crystals for controlling light, only a few recent works $[11,12]$ have considered their role in the formation of dissipative spatial structures. The study of spatial structures in nonlinear periodic systems has important implications not only in nonlinear optics but also in many branches of science, such as biology [13], solid state physics [14], and Bose-Einstein condensates [15].

In this Letter we study the effects of a photonic crystal in the formation of a spontaneous pattern on a nonlinear dissipative system driven out of equilibrium. In particular, we show how the linear phenomenon of photonic band gap affects the selection of a nonlinear spatial structure,
PACS numbers: 42.70.Qs, 05.65.+b, 42.65.Sf, 89.75.Kd

allowing for the complete inhibition of the off-axis emission associated with finite wavelength modulation instabilities (MI).

We consider an optical cavity containing a selffocusing Kerr medium and a linear medium with spatially varying refractive index, i.e., a photonic crystal (see Fig. 1). Both media are antireflection coated. In the mean field approximation, the dynamics of the slowly varying amplitude of the paraxial electric field $E$ can be described by $[16,17]$

$$
\partial_{t} E=-[1+i(\theta+f)] E+i \nabla^{2} E+E_{0}+i|E|^{2} E,
$$

where $\theta$ is the average detuning between the frequency of the pump and the frequency of the cavity, $f$ accounts for the weakly modulated refractive index in the transverse direction of the photonic crystal, $\nabla^{2}$ is the transverse Laplacian, and $E_{0}$ is the input field. All quantities are scaled as in [17].

In model (1) both modulations introduced by spontaneous pattern formation and by the periodic variation of the photonic crystal are described within the paraxial approximation. At difference with the coupled-mode theory, which is limited to spatial structures extended over a large number of photonic crystal wavelengths, Eq. (1) can properly describe transverse structures even smaller than a single period of the photonic crystal. Moreover, while in the tight-binding approximation (discrete) diffraction appears only through the eva-

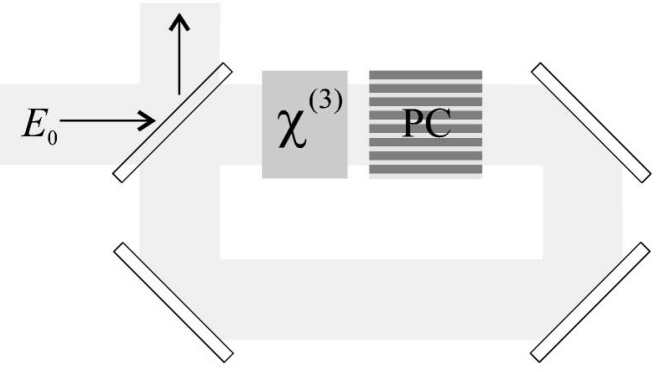

FIG. 1. Ring cavity containing a medium with a cubic nonlinearity $\left(\chi^{(3)}\right)$ and a photonic crystal (PC)slab. $E_{0}$ is the plane wave input field partially transmitted into the cavity. The other mirrors are assumed to be perfectly reflecting. 
nescent coupling between the strongly localized (guided) modes in the photonic crystal, model (1) fully describes diffraction.

In the absence of any modulation of the refractive index, the intensity of the homogeneous steady-state solution $I_{s}$ is implicitly given by

$$
I_{0}=I_{s}\left[1+\left(I_{s}-\theta\right)^{2}\right]
$$

where $I_{0}=\left|E_{0}\right|^{2}$. We consider $\theta<\sqrt{3}$ to avoid homogeneous state bistability. In this case, above a certain threshold $\left(I_{s}=1.0\right)$ a modulation instability takes place, leading to off-axis emission with a critical transverse wave number [18]:

$$
q_{c}=\sqrt{2 I_{s}-\theta} .
$$

In 1D systems, the case considered here, a stripe pattern arises. We study how this mechanism of pattern formation is strongly influenced by the addition of a modulated refractive index.

We recall first the behavior of a simple device consisting of a linear cavity with totally reflecting mirrors. In this case, Eq. (1) reduces to

$$
\partial_{t} E=-i[\theta+f(x)] E+i \nabla^{2} E .
$$

This equation is formally equivalent to the GrossPitaevskii equation with a periodic lattice potential in the limit of noninteracting Bose-Einstein condensate [19]. For $f(x)=0$, the modes $e^{i q x}$ of the cavity are such that $q=\sqrt{-\theta}$ compensates for the cavity detuning [Fig. 2(a)]. However, when the cavity is filled with a photonic crystal, a band gap may appear; i.e., for a given modulation amplitude, there may be detuning values for which no field can propagate in the cavity [see the band gap in Fig. 2(b)].
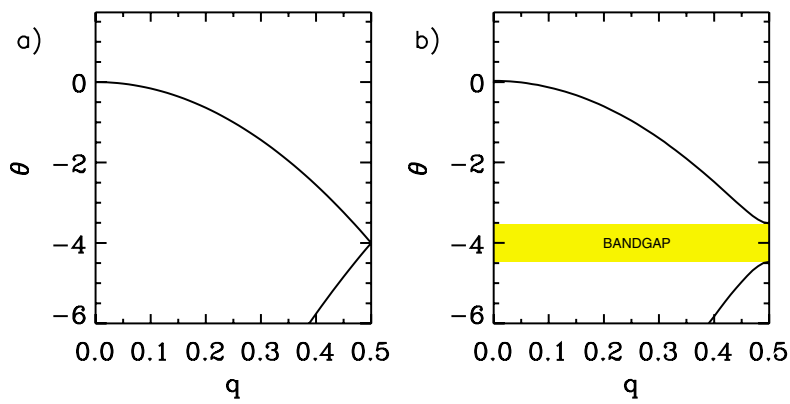

FIG. 2 (color online). (a) Dispersion relation of a perfect linear cavity without photonic crystal $[f(x)=0]$, and (b) with a photonic crystal [we assume $f(x)$ to be a step function between -0.75 and 0.75 of wave number $\left.q_{\mathrm{PC}}=4.0\right]$. The band gap appears for detuning values $-4.47<\theta<-3.51$. For a better comparison, in both cases the calculations have been performed on the basis of Bloch waves in order to reduce the wave number $q$ to the first Brillouin zone. In (a) this results in a reflection of the dispersion relation at $q=0.5$. $q$ is given in units of $q_{\mathrm{PC}}$
Comparing the modes $q$ of the linear cavity and the nonlinear critical wave number $q_{c}$ of Eq. (3), one can see that in both cases off-axis emission compensates for the effective detuning. In particular, the term $2 I_{s}$ in the square root of Eq. (3) accounts for the contribution of the nonlinearity to the detuning. Above the MI threshold, off-axis emission in the nonlinear case may take place not only at the critical wave number but also within a whole band of unstable transverse modes. Figure 3(a) shows the unstable wave numbers $q$ of a nonlinear cavity without photonic crystals for each value of the detuning $\theta$ and $I_{s}=1.12$.

In the presence of the photonic crystal, the linear calculation leading to Fig. 2(b) cannot describe the behavior of the nonlinear cavity. The appearance of a photonic band gap, however, has important consequences for the MI leading to off-axis emissions.

We first find the fundamental solution of the problem with the modulated refractive index (see Fig. 4). This solution is now modulated at the frequency of the photonic crystal. Then, we proceed with a numerical stability analysis [20] of this solution for a pump intensity $12 \%$ above the MI $\left(I_{s}=1.12\right)$, just in the same way as Fig. 3(a) was obtained in the case without photonic crystal. Note, however, that the linear stability analysis of the fundamental solution now requires solving the eigenvalue problem associated to a linear differential operator with periodic coefficients. Then, from the Bloch-Floquet theorem, the eigenmodes can be written in the form of Bloch waves $\psi=M(x) e^{i q x}, M(x)$ being a function with the same periodicity as the photonic crystal. The wave number $q$ of the Bloch functions takes values within the first Brillouin zone of the lattice defined by the wave vectors of the photonic crystal $0<q<0.5 q_{\mathrm{PC}}$. The results are shown in Fig. 3(b) [21]. The presence of the photonic crystal inhibits the pattern forming instability for detuning values $-3.35<\theta<-2.39$. This can be interpreted as follows. For detuning values within this band, the overall effect of the photonic crystal and nonlinearity is to create a photonic band gap for which no extra off-axis emission can
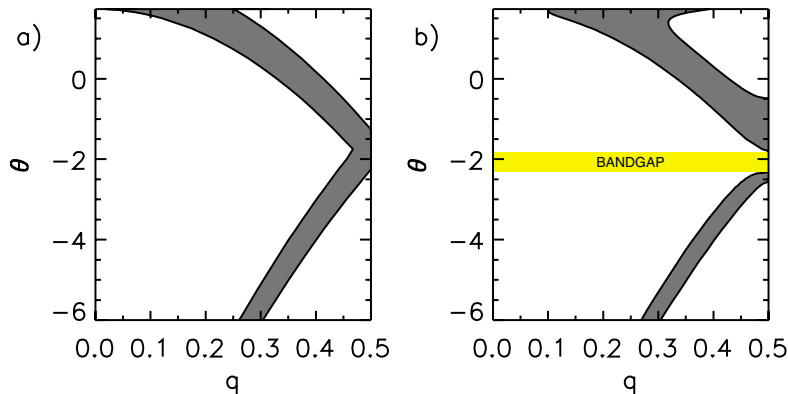

FIG. 3 (color online). (a) The shadowed region indicates the unstable wave numbers of a homogeneous nonlinear Kerr cavity at $12 \%$ above MI threshold $\left(I_{s}=1.12\right)$. (b) The same as in (a) in the presence of the same photonic crystal as Fig. 2(b). For this value of $I_{s}$, the band gap appears for $-2.35<$ $\theta<-1.75$. 

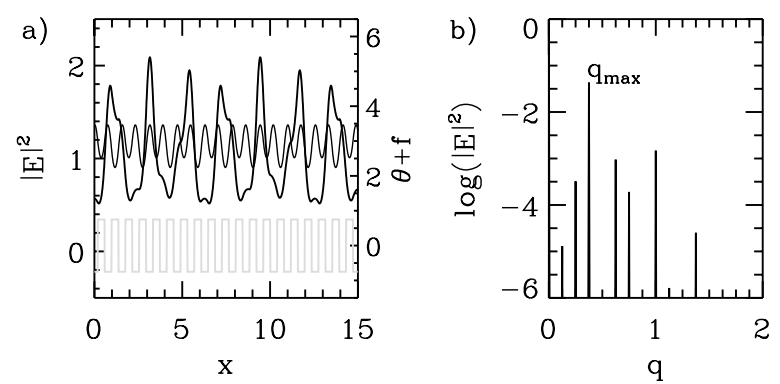

FIG. 4. (a) Intensity of the fundamental (thin black line) and pattern (thick black line) solutions for $I_{s}=0.99$ and $I_{s}=1.12$, respectively $(\theta=0)$. The grey line is the total detuning given by the cavity and the photonic crystal. (b) Far field of the pattern solution. The main wave number of the pattern $q_{\max } \sim$ $q_{c}$ is within the band of unstable modes of the fundamental solution [Fig. 3(b)]. $q$ is given in units of $q_{\mathrm{PC}}$. The large peak in $q=1.0$ corresponds to the spatial frequency of the photonic crystal. The simulations have been performed on a 2048 grid with $\Delta x=0.0122$ and $\Delta t=0.002$ starting from random initial conditions.

take place. Inside the band gap, the fundamental solution regains stability. Outside the band gap, the fundamental solution is unstable, leading to the formation of a high amplitude pattern at the most unstable wave number predicted by the stability analysis [see the thick line curve in Fig. 4(a)]. The final spatial structure has a combined periodicity of the pattern that would appear without photonic crystal $\left(q_{\max }=1.5\right)$ and of the photonic crystal $\left(q_{\mathrm{PC}}=4.0\right)$. The other spatial frequencies are the result of nonlinear interactions and resonances.

The modes of the linear cavity represented in Fig. 2(b) and the unstable transverse modes of the nonlinear cavity shown in Fig. 3(b) have a very different nature. In the first case they are the only allowed modes of emission, while in the second case they are the result of a MI due to the presence of the nonlinearity, diffraction, and dissipation. In particular, while in the first case the band gap means no field inside the cavity, in the driven dissipative cavity the field is always different from zero, but the transverse emission due to the modulation instability is forbidden. In the latter case, nonlinearity may completely modify the band structure when changing the intensity of the input field. For example, the band gap is broader for lower
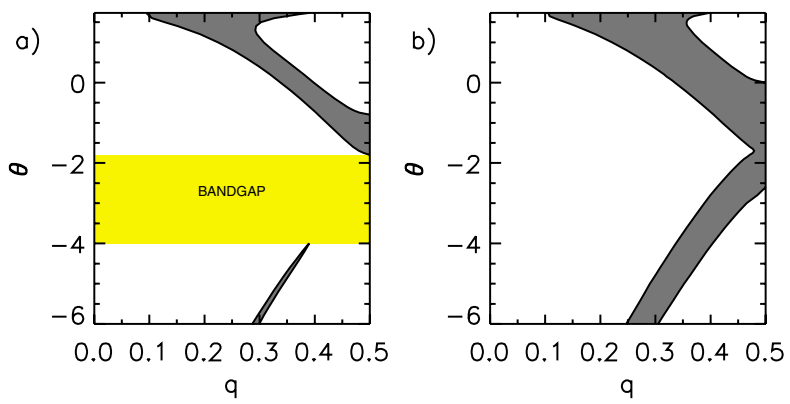

FIG. 5 (color online). The same as Fig. 3(b) for (a) $I_{s}=1.05$ and (b) $I_{s}=1.25$. Note the absence of photonic band gap in (b).

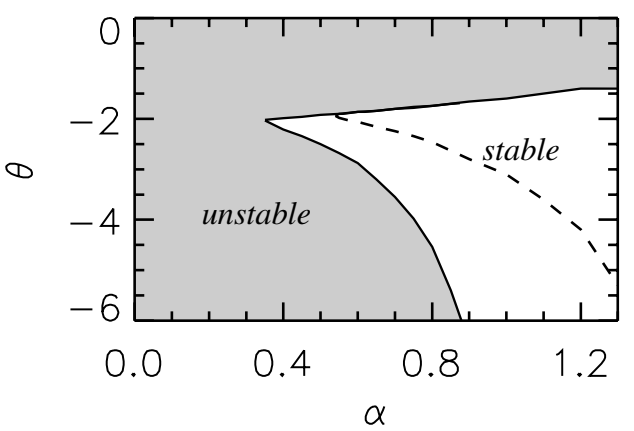

FIG. 6. Instability diagram of the fundamental solution for $I_{s}=1.05$. The fundamental solution is unstable in the shadowed region. The dashed line shows the boundary of the stable region for $I_{s}=1.12$.

values of the input intensity and smaller for larger values, eventually disappearing for high enough input intensities (Fig. 5). In Fig. 6 we also show the band-gap width versus the amplitude $\alpha$ of the modulation. The presence of the modulated medium opens a gap, i.e., an entirely new stable region, in the $\theta-\alpha$ plane.

The inhibition of MI due to photonic crystals can then be used to control the transverse properties of light in nonlinear optical cavities in a way analogous to light control in holey waveguides. For example, we consider a profile of the refractive index represented by the grey line in Fig. 7 and formed by a plateau surrounded by regions of modulation. We see how the large amplitude pattern is inhibited in the modulated regions where the system is anchored to the fundamental solution, while a strong stripe pattern is observed in the plateau (Fig. 7). The wave number of the pattern corresponds to the most unstable wave number of a homogeneous nonlinear cavity. The size of the defect can be reduced down to one and a half wavelengths of the photonic crystal, thus creating a localized mode in the transverse plane (see Fig. 8). Note that in Fig. 8, where a larger amplitude of the modulation of the photonic crystal has been introduced, the inhibition of the MI takes place also for very large intracavity intensity (e.g., $80 \%$ above the MI threshold). A single peak of the large amplitude pattern towers over the

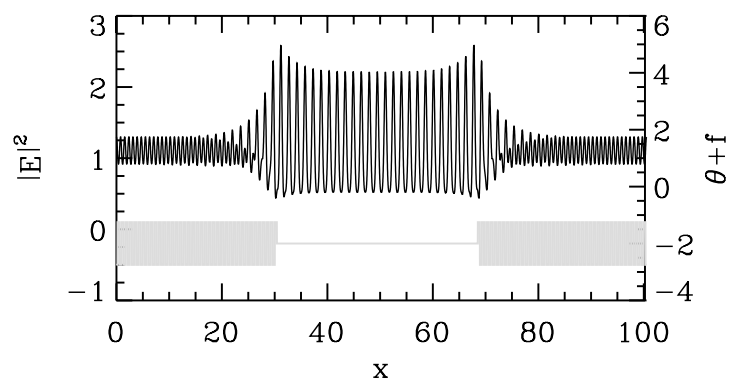

FIG. 7. Pattern formed in a 72 photonic crystal wavelengths long defect for parameter values inside the band gap of Fig. 3(b) $\left(I_{s}=1.12, \theta=-2\right)$. Here we take 8192 grid points. The pattern formed into the defect has a wave number close to the most unstable wave number of a homogeneous nonlinear cavity. 


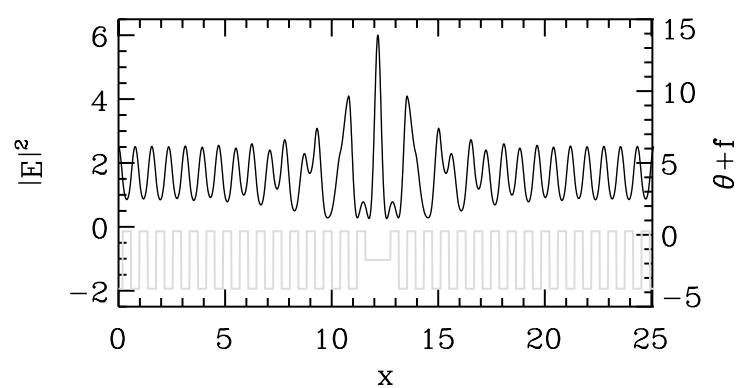

FIG. 8. Localized structure formed inside a defect 1.5 photonic crystal wavelengths long. In this figure, $f(x)$ is a step function between -2.0 and $2.0, \theta=-1.75$ and $I_{s}=1.8$. For these parameter values the band gap is found for detuning values $-2.0<\theta<-0.38$.

fundamental solution in the inhibited zone and signals the presence of the photonic crystal defect. At difference with localization of light in normal photonic crystals, these effects are intrinsically nonlinear since light can propagate outside the defect while nonlinear pattern formation is confined to the defect region. High contrast between the localized peak and the background can be used to diagnose small defects present in a photonic crystal structure.

We have demonstrated that a photonic crystal in a nonlinear cavity can inhibit a pattern formation instability on a scale close to, but different from, that of the modulated refractive index. An important application of this effect is in the case of subcritical patterns where the delaying of the MI may dramatically increase the region of coexistence between the fundamental and patterned solutions. The immediate benefit is to increase the region of existence of cavity solitons [2], thus simplifying their utilization in information processing. We have also characterized a novel localization of light due to defects in the photonic crystal structure and MI inhibition and suggested it as a diagnostic tool of photonic crystals. This light localization can also be used to obtain peculiar output intensity structures by engineering defects in a photonic crystal. Further investigations in these directions and in the two transverse dimensional case are in progress.

Finally, we would like to mention that the phenomenon described here, namely, the inhibition of a modulation instability by a transverse photonic band gap, is not confined to dissipative systems but can be of interest in conservative systems, such as Bose-Einstein condensates - where a modulation instability [22] might be controlled by the addition of an optical lattice - or in light propagation through nonlinear periodic media $[10,23]$.

We thank W. J. Firth for useful discussions. We acknowledge financial support from EPSRC (GR/S28600/ 01, GR/S03898/01, and GR/R04096) and the European project QUANTIM. G.-L. O. acknowledges support from SGI and the Royal Society-Leverhulme Trust.
[1] M. C. Cross and P. C. Hohenberg, Rev. Mod. Phys. 65, 851 (1993); D. Walgraef, Spatio-temporal Pattern Formation (Springer, Berlin, 1997).

[2] W. J. Firth and C. O. Weiss, Opt. Photonics News 13, No. 2, 54 (2002); S. Barland et al., Nature (London) 419, 699 (2002).

[3] J. D. Joannopoulos, R. D. Meade, and J. N. Winn, Photonic Crystals (Princeton University Press, Singapore, 1995); J. D. Joannopoulos, P. R. Villeneuve, and S. Fran, Nature (London) 386, 143 (1997).

[4] E. Yablonovitch, Phys. Rev. Lett. 58, 2059 (1987); S. John, Phys. Rev. Lett. 58, 2486 (1987).

[5] J. C. Knight, Nature (London) 424, 846 (2003).

[6] S. Mingaleev and Y. Kivshar, Opt. Photonics News 13, No. 7, 48 (2002); V. Berger, Phys. Rev. Lett. 81, 4136 (1998).

[7] Nonlinear Photonic Crystals, edited by R. E. Slusher and B. J. Eggleton (Springer, Berlin, 2003).

[8] Y.S. Kivshar and G. P. Agrawal, Optical Solitons: From Fibers to Photonic Crystals (Academic Press, San Diego, 2003).

[9] F. Lederer, S. Darmanyan, and A. Kobyakov, in Spatial Solitons, edited by S. Trillo and W. Torruellas (Springer, Berlin, 2001), p. 269; D. N. Christodoulides, F. Lederer, and Y. Silberberg, Nature (London) 424, 817 (2003).

[10] F. Abdullaev, S. Darmanyan, and J. Garnier, Prog. Opt. 44, 303 (2002).

[11] U. Peschel, O. Egonov, and F. Lederer, FriedrichSchiller-University Jena, report, 2003 (to be published).

[12] K. Staliunas, Phys. Rev. Lett. 91, 053901 (2003).

[13] J. E. Curtis, B. A. Koss, and D. G. Grier, Opt. Commun. 207, 169 (2002).

[14] See, for instance, review articles in a special issue on Nature Insight: Photonic Technologies, edited by L. Venema [Nature (London) 424, 809 (2003)].

[15] M. Greiner et al., Nature (London) 415, 39 (2002).

[16] L. A. Lugiato and R. Lefever, Phys. Rev. Lett. 58, 2209 (1987); W. J. Firth et al., Phys. Rev. A 46, R3609 (1992).

[17] L. A. Lugiato and C. Oldano, Phys. Rev. A 37, 3896 (1988). Typically, for a cavity a few $\mathrm{cm}$ long and beams a few mm wide, MI takes place at input powers around $10 \mathrm{~mW}$, which is easily reachable in standard experimental configurations.

[18] A. J. Scroggie et al., Chaos Solitons Fractals 4, 1323 (1994).

[19] P. J. Y. Louis et al., Phys. Rev. A 67, 013602 (2003).

[20] G. Harkness et al., Phys. Rev. E 66, 046605 (2002); D. Gomila and P. Colet, Phys. Rev. E 66, 046223 (2002); Phys. Rev. A 68, 011801(R) (2003).

[21] Note that for each $\theta$ one has to change $E_{0}$ in order to keep $I_{s}$ fixed. In spite of the detuning modulation, we guarantee to be somehow above MI since there are unstable wave vector bands both above and below the band gap.

[22] K. E. Strecker et al., Nature (London) 417, 150 (2002); K. Berg-Sørensen and K. Mølmer, Phys. Rev. A 58, 1480 (1998); V.V. Konotop and M. Salerno, Phys. Rev. A 65, 021602(R) (2002).

[23] A. A. Sukhorukov and Y. S. Kivshar, Phys. Rev. E 65, 036609 (2002); A.V. Yulin, D.V. Skryabin, and W. J. Firth, Phys. Rev. E 66, 046603 (2002). 